\title{
Innovaciones técnicas, estilísticas y temáticas en la cerámica de los Zuloaga
}

\author{
A. RUBIO CELADA \\ Universidad Complutense de Madrid. Ciudad Universitaria. Madrid
}

\begin{abstract}
La cerámica de los Zuloaga abarca varias generaciones, desde los años setenta del siglo XIX. Los primeros son Guillermo, Germán y Daniel, a los que su padre, Eusebio, arcabucero de Isabel II y director de la Real Armería, manda a estudiar a la Escuela de Cerámica de Sèvres. A ellos se deberá el resurgir de la fábrica de la Moncloa. Los Zuloaga recuperarán las técnicas cerámicas tradicionales, como la cuerda seca, la cuenca y el reflejo metálico, introduciendo los estilos de moda en Europa como el Neorrenacimiento y el Modernismo. En 1893 Daniel Zuloaga se establece en la Fábrica de Vargas en Segovia, instalando a principios de siglo su propio taller, en la iglesia románica de San Juan de los Caballeros. Aquí, es ayudado por sus hijos Juan, Esperanza y Teodora, incorporando nuevos estilos como el neorrománico y el regionalista, con temas sacados de los tipos y costumbres castellanas, dentro de la estética de la Generación del 98. En su taller la investigación cerámica fue una constante, llegando a desarrollar técnicas originales como los esmaltes sobre pizarra, o la aplicación de la fotografía a la cerámica.
\end{abstract}

Palabras clave: Cerámica artística, historia cerámica, cerámica tradicional

Technical, stylistic and thematic innovations in the Zuloaga's ceramics

The Zuloagas' ceramics covers several generations, since the 1870's. The first artists in the family were Guillermo, Germán and Daniel. Their father, Eusebio, arquebusier of Queen Isabel II and Chief of the Royal Gunsmith's House, sent them to study to the school of ceramics in Sévres, France. The resurgence of the factory of La Moncloa, in Madrid, was possible thanks to them. The Zuloagas restored the traditional techniques in ceramics; for example, the one of "cuerda seca" or "cuenca"-glazed and lustre painted ones, introducing the styles that were on fashion in Europe: Neo-Renaissance and Modernism. In 1893, Daniel Zuloaga settled into the Vargas factory in Segovia, and he opened, at the beginning of the XIX century his own workshop in the church of San Juan de los Caballeros, of Romanesque style, placed in the centre of this town, Segovia. Here he was helped by his son and daughters, Juan, Esperanza and Teodora, adopting new trends in art, like the Neo-Romanesque style and the Regionalist style, with themes he took from Castilian models and customs, within the aesthetic lines of the 98's Generation.In his workshop he constantly investigated into ceramics and he even developed original techniques, like glazing on slate or applying photographs to ceramics.

Key words: Artistic ceramic, ceramic history, traditional ceramics.

\section{INTRODUCCIÓN}

La presente ponencia está en relación con la tesis doctoral que actualmente se realiza en la Universidad Complutense de Madrid con el siguiente título: “De la tradición a la Modernidad. Los Zuloaga ceramistas".

De todos es conocido el apellido Zuloaga, pero, por regla general se asocia con el pintor Ignacio y no con el mundo de la cerámica. En el caso de que sí se relacionara con las artes del barro, sólo el nombre de Daniel es bien conocido, quedando eclipsado el resto de la familia.

La cerámica de los Zuloaga se desarrolla entre el último tercio del s. XIX y durante todo el s. XX. En este largo período de tiempo se suceden tres generaciones de ceramistas:

- De los años 70 a los 90 del s. XIX trabajan los hermanos Guillermo, Germán (muere en 1886) y Daniel, identificándose este primer período con la fábrica de la Moncloa en Madrid.

- De 1893 a 1906 trabaja Daniel en la fábrica de Vargas en Segovia, y en 1907 en la fábrica de Pasajes en Guipúzcoa.

- De 1908 a 1921, en que muere Daniel, éste trabaja en compañía de sus hijos Juan, Teodora y Esperanza, conociendo su cerámica un período de esplendor y reconocimiento de los intelectuales del momento, que visitan su famoso taller dentro de la iglesia románica de San Juan de los Caballeros en Segovia.

- De 1921 a 1947 sigue la producción del taller de San Juan, en la línea de Daniel Zuloaga, repitiendo los mismos modelos, fundamentalmente. A partir de este año la Iglesia se convierte en Museo y Escuela de cerámica, siendo los profesores los Zuloaga.

- Daniel Zuloaga Olalla, hijo de Juan, en su propio taller, sigue realizando cerámicas en la tradición heredada de su abuelo, y con los mismos moldes.

El comienzo en el mundo de la cerámica de los Zuloaga, coincide con un momento de esplendor de las Artes Decorativas en toda Europa.

La segunda mitad del s. XIX supone una mirada al mundo de la artesanía, a consecuencia del auge que estaba tomando la industria, con la desaparición de los oficios tradicionales, y el abandono del campo para buscar trabajo en las fábricas de las ciudades. 
En Europa se desarrollan movimientos que quieren recuperar las técnicas artesanales, tomando como paradigma el mundo de la Edad Media, tal como ocurre en Inglaterra con las "Arts and Crafts".

En España, aunque estos movimientos no tienen tanta importancia, sí hay arquitectos que potenciarán la artesanía en sus edificios, tal es el caso de Gaudí en Cataluña, o de Velázquez Bosco en Madrid. Este último tiene gran importancia, ya que utilizará frecuentemente la cerámica aplicada a la arquitectura, siendo un destacado representante de la denominada "arquitectura de color", recurriendo para ello a los ceramistas Zuloaga: Guillermo, Germán y Daniel.

\section{LA ÉPOCA DE LA MONCLOA}

Los hermanos Zuloaga, gracias a su padre Eusebio, arcabucero de Isabel II y director de la Armería Real, recibirán un aprendizaje sólido como ceramistas en Sèvres ${ }^{1}$. Allí se pondrán al día sobre las corrientes que imperan en Europa en esos momentos: Orientalismo, neorrenacimiento,... así como en las técnicas usadas en mayólica, que desplazará a la porcelana, siendo Palissy uno de los más imitados.

Aquí aprenderán a usar pastas blancas caolínicas, cocidas a $1200^{\circ} \mathrm{C}$, y técnicas pictóricas bajo baño, que considerarán como la verdadera cerámica, frente a la cocida por debajo de $1000^{\circ} \mathrm{C}^{2}$.

De vuelta a España, y gracias a su padre nuevamente, conseguirán volver a poner en marcha la antigua fábrica de la Moncloa, formando una sociedad en 1881, en la que participan personajes importantes como el conde Morphy, secretario del rey y el Ministro de Instrucción Pública.

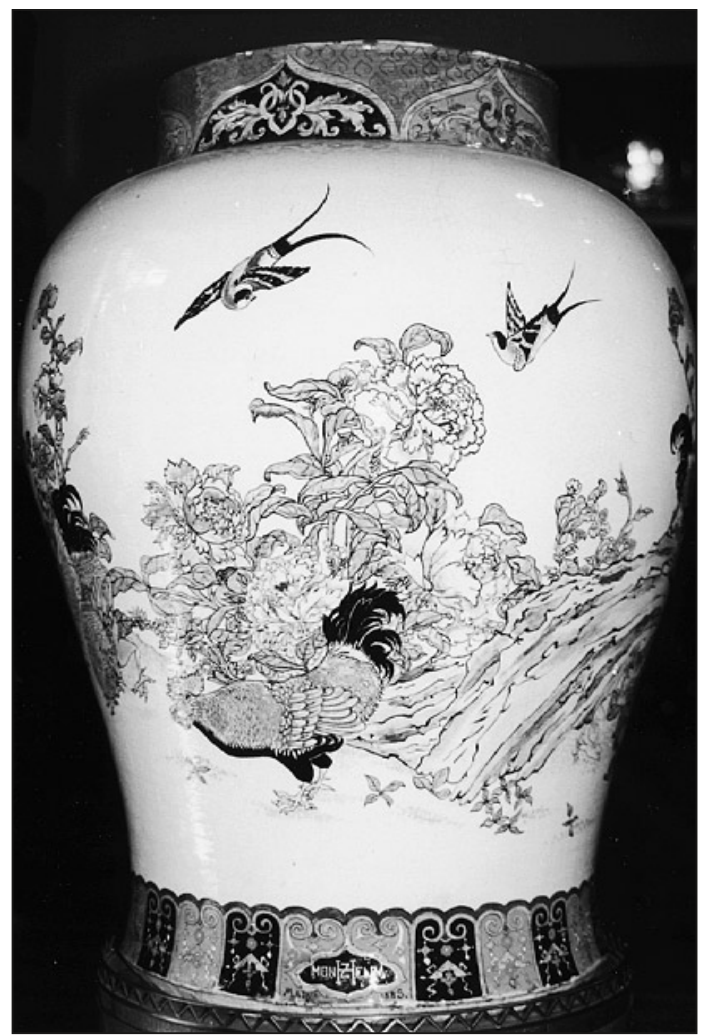

Fig. 1. Tibor con decoración inspirada en porcelanas chinas del s. XVIII. Hermanos Zuloaga. Fábrica de la Moncloa. 1883.
Será en los trabajos que la Moncloa realiza para el Pabellón de la Exposición Minera del Retiro de 1883 donde se puedan ver sus primera cerámicas ${ }^{3}$.

Entre las técnicas recuperadas, basadas en las tradición hispano musulmana, está la cuerda seca, que es utilizada en el Palacio de Velázquez, según $\mathrm{M}^{\mathrm{a}}$ Jesús Quesada4 ${ }^{4}$ Para Antonio Perla “...los motivos están contorneados por una línea de manganeso... cuya función es la de reforzar la imagen, no la de contener espacios de color ... no estamos ante una cerámica de cuerda seca como en ocasiones se ha afirmado..." ${ }^{5}$. Una precisión más sobre esta técnica utilizada en el Palacio de Velázquez, a raíz de su restauración, es “...ha sido realizada con esmaltes muy fluidos, utilizando un perfilado de manganeso... para reforzar las líneas del dibujo, todo ello bajo una cubierta plúmbea" 6 .

Otra técnica empleada es la de cuenca, en el exterior del edificio. Asimismo se pintan escenas sobre cubierta blanca estannífera, en un estilo neorrococó, mientras que para cuerda seca y cuenca se ha preferido el neorrenacimiento. También se utiliza el relieve en medallones sobre las enjutas, siguiendo el estilo renacentista de los Della Robbia.

En cuanto a las pastas, parece que a cargo de Guillermo, se utilizan tanto rojas como blancas, de base caolínica, ensayándose con tierras de Aragón y de Toledo. Hay que remarcar que se utiliza una base blanca de estaño sobre una pasta blanca, en lugar de aprovechar el color de la base.

La restauración del Palacio de Velázquez permite conocer la estructura de algunos azulejos, que presentan por el reverso 64 cuadrados rehundidos, realizados por el sistema de prensa, y cuya función es ayudar mecánicamente al agarre del mortero, su superficie es de $17,5 \mathrm{~cm}$. de lado y un grosor de $1,5 \mathrm{~cm} .^{7}$.

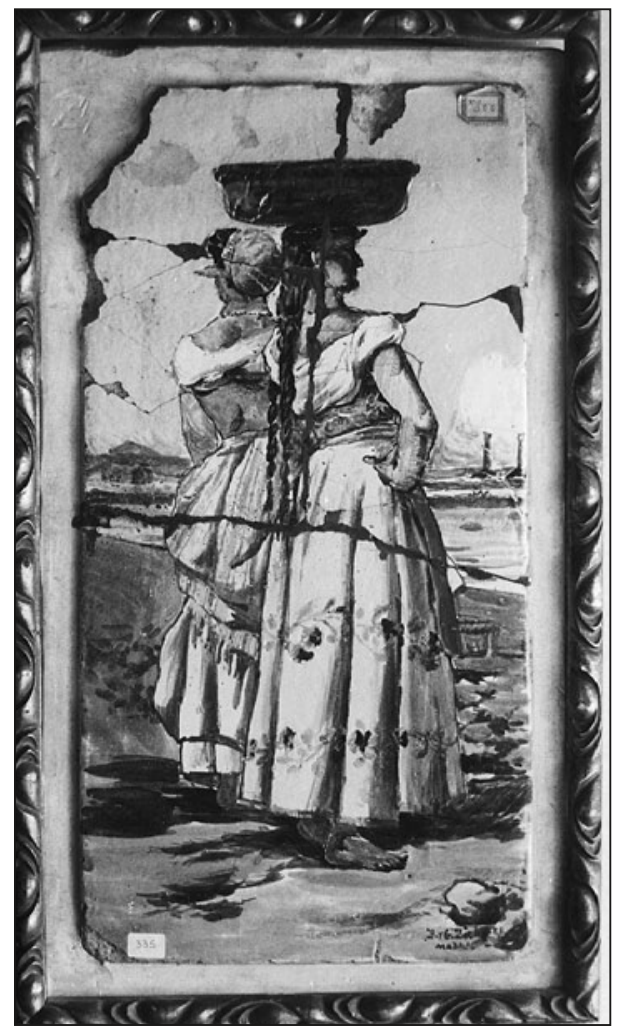

Fig. 2. Placa con decoración de pescadoras. Daniel y Guillermo Zuloaga. Finales de los años 80 del s. XIX. 
Daniel Zuloaga, ya que no la realizó por encargo, sino por su propio gusto. En ella destaca, en grandes letras capitales negras, la palabra "LABORATORIO", sobre un fondo ajedrezado azul celeste y blanco.

La fachada adopta una forma rectangular, rematada en piñón triangular. Presenta como decoración una cartela ovalada con marco de cueros recortados, a cada lado de la puerta, donde se inscriben los retratos, en grisalla de azul cobalto bajo cubierta, del ceramista Bernard Palissy a la izquierda y del químico Lavoisier a la derecha, simbolizando respectivamente el arte y la ciencia, de cuya unión nacería la cerámica. Estas cartelas se inscriben en sendos motivos de candelieri con intrincados roleos y grutescos al más puro estilo renacentista.

Los elementos decorativos están pintados bajo baño transparente a gran fuego, separados por la técnica del entubado, que consiste en una línea en relieve con pasta líquida, con la que se ha realizado a mano todo el dibujo. He aquí otra innovación en la cerámica de los Zuloaga.

Abajo aparece un zócalo con una fila de azulejos rectangulares en relieve, con decoración de roleos, con un barniz transparente que deja ver la pasta blanca y con reflejos metálicos perfilando los motivos. Arriba y abajo de estos, otra fila de azulejos de formato muy estrecho con decoración vegetal, realizada a cuenca.

En este "LABORATORIO" es donde aprendió cerámica Joaquín Castellarnau, y donde realizó con Daniel experimentos en fotocerámica. Es ésta una de las técnicas cerámicas de las que no existe constancia que haya realizado ningún otro ceramista en España, a finales del siglo XIX y principios del siglo XX.

Según Juan Zuloaga y su cuñado Mazorriaga, Castellarnau “...junto con Zuloaga hizo multitud de pruebas para aplicar la

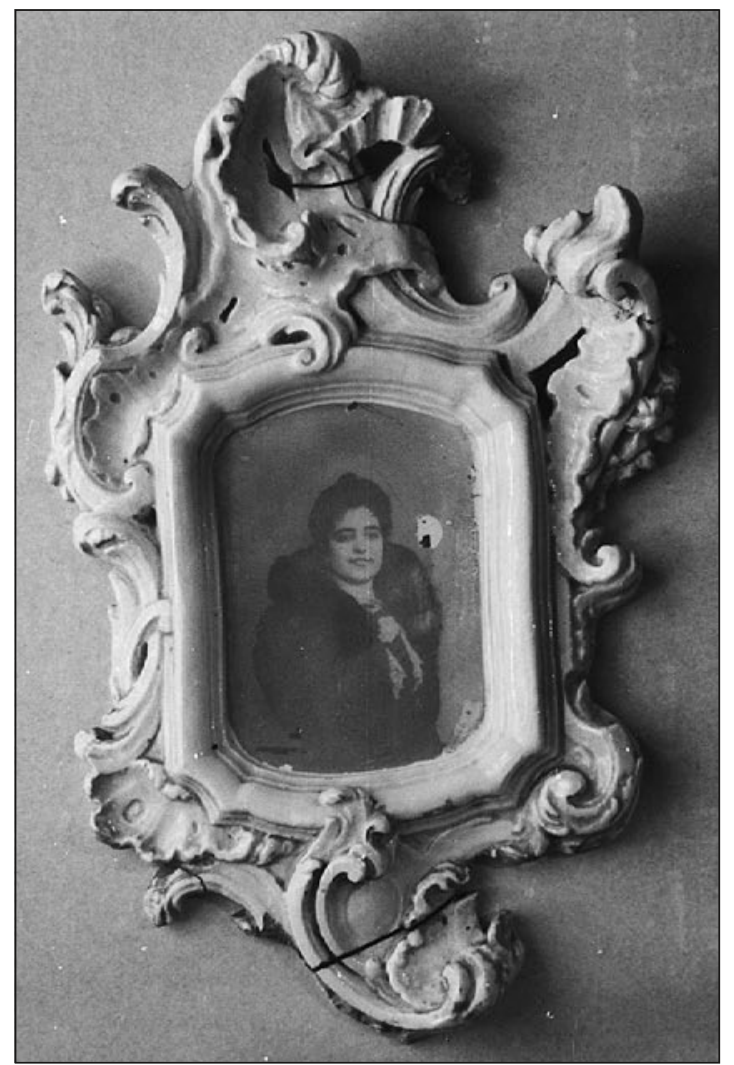

Fig. 4. Fotocerámica. Daniel Zuloaga y Joaquín Castellarnau en el Taller de Daniel en la fábrica de Vargas (1893-1906). fotografía a la cerámica -con el éxito más halagador- por un procedimiento muy complicado que consistía en pasar la prueba fotográfica sobre el bizcocho y luego fijarla con un baño transparente a gran fuego.

Cándida Zuloaga tiene un retrato en coloración rojiza realizado por este método, que está perfectamente logrado" 21 .

Esta fotocerámica se ha realizado en el interior de una cornucopia, siguiendo los modelos alcoreños del XVIII, copiados en la Tercera Época de la Moncloa (Figura 4) ${ }^{22}$ la materia es pasta blanca artificial, del tipo utilizado en la fábrica de Vargas, cocida a $1200^{\circ} \mathrm{C}$. El procedimiento utilizado en la técnica de la fotocerámica se relaciona con la fotomecánica. Una vez realizado el negativo difiere de ésta en que la tinta de impresión es sustituida por bicromatos, sales de hierro, etc. disueltas en un medio graso ${ }^{23}$. Así el retrato bajo barniz transparente está listo para ser cocido a alta temperatura.

Estos experimentos de Castellarnau y Daniel Zuloaga es posible que correspondan a los últimos años de la época de Vargas, y que sea el traslado de Daniel a Pasajes lo que no permitiera desarrollar esta técnica.

En la fábrica de Vargas será donde Daniel realice sus primeros diseños modernistas, estilo poco conocido, ya que no se manifiesta tanto en fachadas, como en interiores o piezas de pequeño formato ${ }^{24}$. Éste es el caso de un portal madrileño, en la c/ Barquillo, donde con el arquitecto Jesús Carrasco realiza en 1900 todo una iconografía de bustos modernistas, inspirados en Mucha (Figura 5) ${ }^{25}$. La materia es pasta blanca artificial, y la técnica decorativa es azul cobalto bajo baño a gran fuego, y reflejo metálico dorado en tercera cocción. Este busto aparece también en la orla que rodea al mural de los pavos del Museo Zuloaga, n1 inv. 192.

De estilo modernista, también en el Museo Zuloaga, en el jarrón

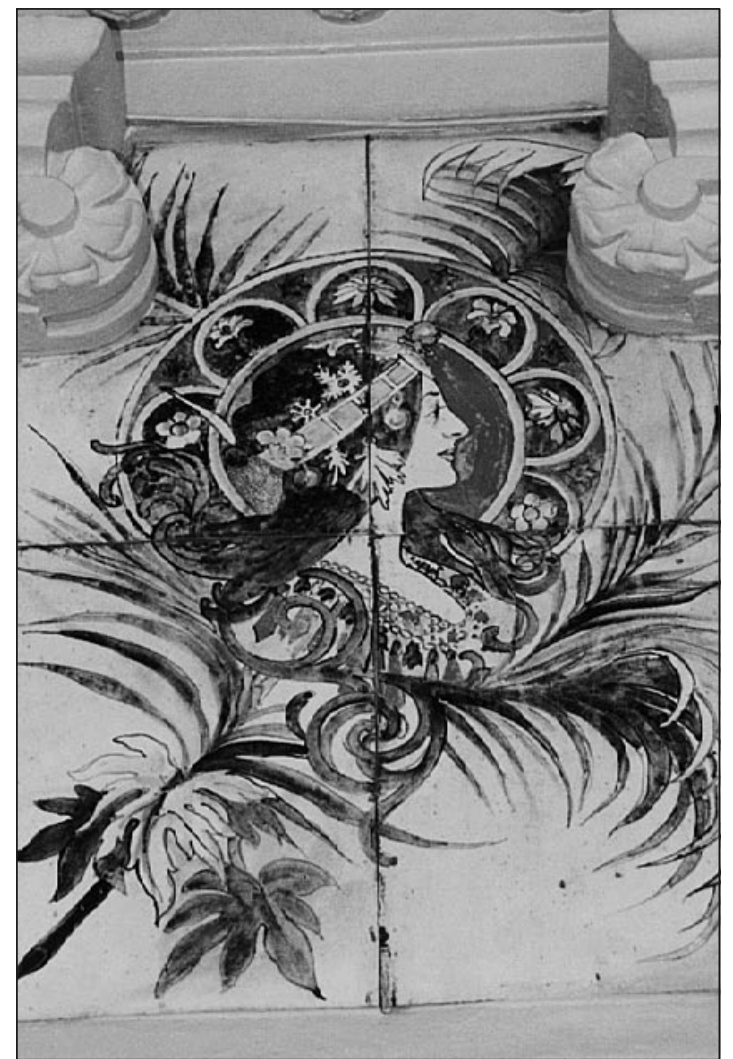

Fig. 5. Panel modernista formado por cuatro azulejos con decoración de una cabeza femenina de perfil, en azul cobalto y dorado. Copiada de Mucha por Daniel Zuloaga. 1900. 
decorado con un pavo, con la técnica del entubado, n1 inv. 557.26

De la época de Vargas es una de las pocas obras importantes que Daniel Zuloaga realizó en Segovia. Se trata del Altar del Cristo de Lozoya de la Catedral de Segovia, donde en estilo neorrenacentista, tipo Della Robbia, desarrolla una serie de técnicas diversas, algunas poco utilizadas como las pastas coloreadas ${ }^{27}$. Otras, como el reflejo metálico, aparecen por primera vez, según $\mathrm{M}^{\mathrm{a}}$ Jesús Quesada, en este tipo de obras.

\section{LA ÉPOCA DE SAN JUAN DE LOS CABALLEROS}

Tras su ruptura con la Fábrica de Vargas, Daniel se traslada a la Fábrica de Pasajes en Guipúzcoa, donde permanecerá un año, volviendo tras el fracaso de nuevo a Segovia, donde deberá empezar desde cero. Gracias a un pequeño horno de una sola pieza, que había comprado a su hermano Guillermo, realizará algunas obras con la técnica de sobrecubierta y temperatura no superior a $1000^{\circ} \mathrm{C}$, como la Chimenea del Casino de la Unión en Segovia ${ }^{28}$.

Instalado en San Juan de los Caballeros, Daniel debe afrontar la búsqueda de materiales, ya que ahora no dispone del caolín de Vargas. Poco a poco, irá dejando las pastas blancas y trabajando con rojas. Para sus cuadros cerámicos no duda en recurrir a todo tipo de azulejos de fábricas españolas y extranjeras, por lo que nos encontramos con todo tipo de formatos, acabando por usar en el taller, sobre todo posteriormente sus hijos, las losetas rojas de gres catalán. Estos, Esperanza, Teodora y sobre todo, su hijo Juan, le serán de gran ayuda.

Se puede decir que San Juan se caracterizó, no sólo por la repetición de modelos, sino por un espíritu de investigación,

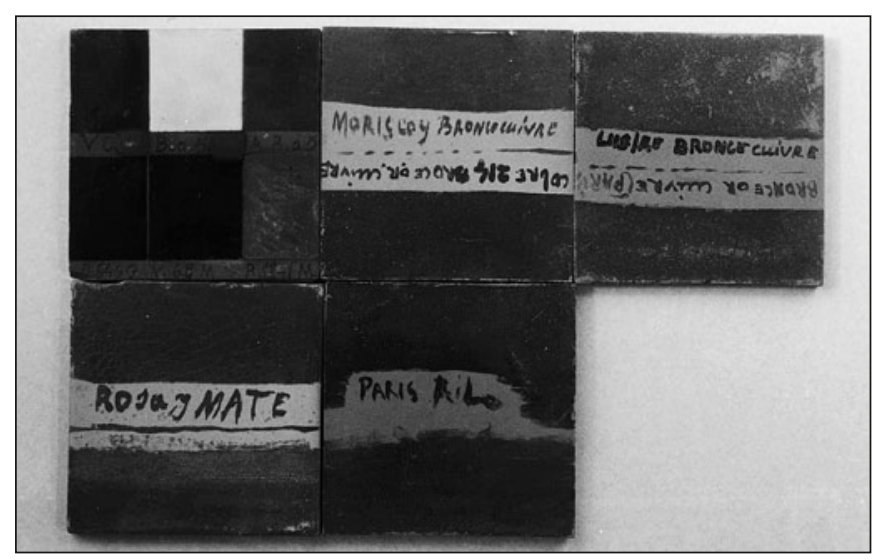

Fig. 6. Pruebas de reflejos metálicos. Taller de los Zuloaga en San Juan de los Caballeros. S. XX.

encontrando en los años 20 y 30, pruebas de pastas y esmaltes, a pesar de la larga experiencia del taller. Como ejemplo, he aquí unas fórmulas ${ }^{29}$ :

\section{"Pasta de porcelana"}

Arcilla de caolín ..................................................................... 43,5

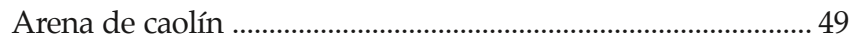

Arena Bernuy .......................................................................... 4,

Carbonato de cal .................................................................... 4,5

Azul cobalto (2)

Oxido cobalto …….................................................................... 0,700

Fundente .......................................................................... 9,300"

Dada la escasez de noticias sobre los materiales utilizados por

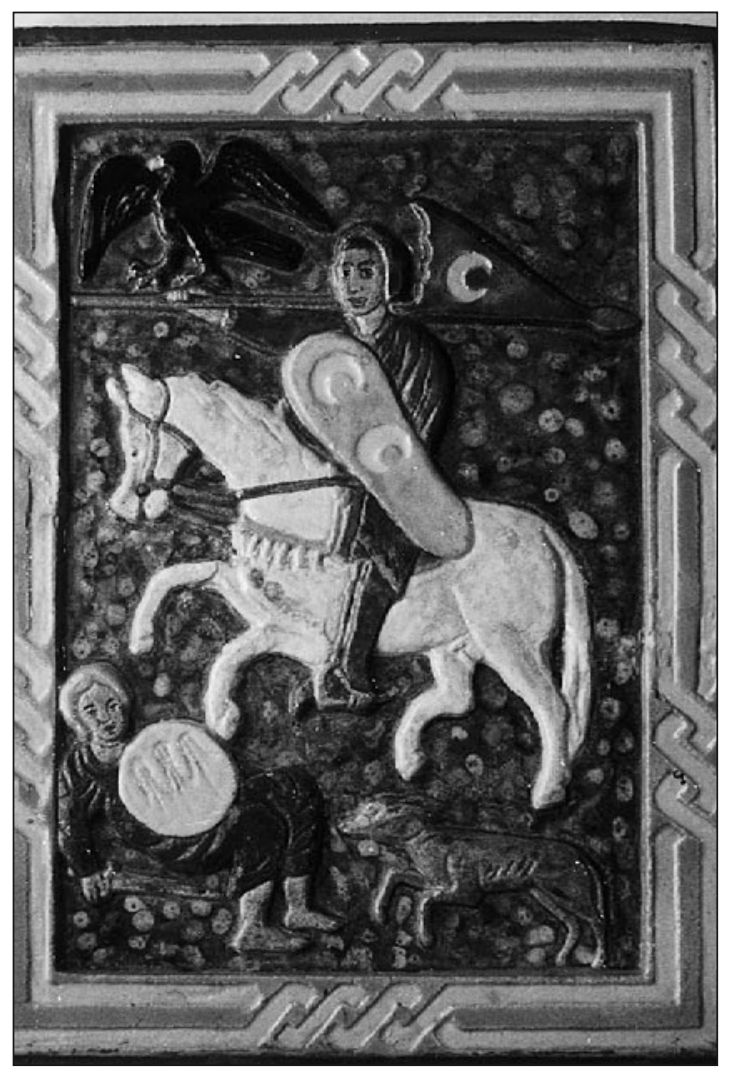

Fig. 7. Placa con decoración en relieve, copiada de una pintura mural de la Torres de Hércules en el convento de das Dominicas (Segovia). Juan Zuloaga. S. XX.

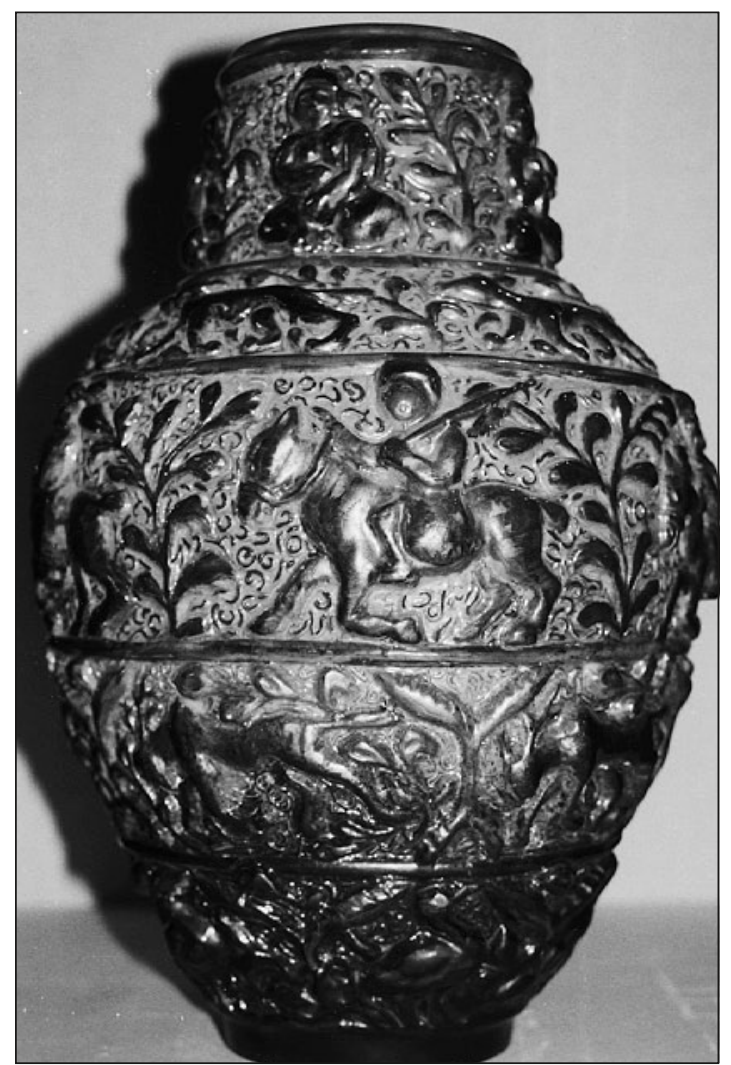

Fig. 8. Jarrón del Taller de los Zuloaga. Copia de una cerámica persa del s. XIII. Taller de San Juan de los Caballeros. S. XX. 
los Zuloaga, estas fórmulas tienen un especial interés ${ }^{30}$.

Del año 1934, de la memoria presentada por Juan Zuloaga al Concurso Nacional de Arte Decorativo tenemos una minuciosa descripción de los materiales y técnicas empleados: caolín de Torrijos en Toledo, feldespato y cuarzo de Segovia. Colores bajo baño, conseguido con sílice de Bernuy de Porreros en Segovia, sosa, potasa de Alicante, minio, albayalde, bórax y ácido bórico. Estos materiales están cocidos a 1100 grados.

El resultado obtenido de la combinación de los materiales anteriores fue la obtención del Primer Premio. Se puede ver esta cerámica, que se encuentra en los depósitos del Museo de Artes Decorativas de Madrid, n1 inv. $16617^{31}$.

Gracias a Juan, se conoce muchos de los procedimientos cerámicos del taller de San Juan, no sólo por las memorias minuciosas que hace acompañando las piezas que manda a los concursos, sino por diversas anotaciones y escritos conservados en el Museo Zuloaga ${ }^{32}$.

En esta Tercera Época del Taller de San Juan, aparecerán temas nuevos que se desarrollarán con técnicas ya vistas, como cuerda seca o cuenca, adquiriendo más importancia los reflejos y lustres, que a veces cubren por completo toda la pieza, con infinidad de matices. Se conservan azulejos de pruebas para reflejos (Figura 6) ${ }^{33}$.

Entre los temas más repetidos ahora, se encuentran los inspirados en el románico, sin duda debidos no sólo a la influencia de la iglesia románica de San Juan en la que trabajan, sino también a la riqueza de este estilo en la ciudad de Segovia y provincia.

Uno de estos temas románicos son las pinturas de la Torre de Hércules del Convento de las Dominicas en Segovia (Figura 7). Una de ellas presenta una escena bélica, que se ha reproducido en una placa, excavando el fondo. El material presenta un aspecto granular, parecido a piedra arenisca.

Otro tema es el de la cerámica persa de Rhagés, copiando formas y motivos de esta cultura, a veces, de forma literal, como este jarrón, hecho a molde, y que presenta una decoración en relieve, dispuesta en fajas horizontales (Figura 8).

Otra faceta en la obra de Daniel es la relación que mantuvo con intelectuales y artistas del momento, que le visitaron frecuentemente en su taller de San Juan, y que convirtieron a Segovia en centro de cultura. En primer lugar, destaca su sobrino Ignacio, con el que compartió muchas vivencias. Otro artista con el que colaboró, fue el escultor Sebastián Miranda, con el que realizó pequeñas figuras de cerámica. Citaremos por último, al fotógrafo Luis Ocharán, al que ayudó en la realización de sus famosos fotos del Quijote. Daniel utilizará estas fotos en tapices y cerámicas, como el jarrón conocido como "tibor Sancho", realizado a molde, con la técnica de cuenca (Figura 9).

Para terminar se cita uno de los temas más representativos y más reproducidos del taller de San Juan, tanto por los hijos de Daniel como por los operarios. Se trata de los tipos y escenas regionalistas, sobre todo segovianos, llamados de "catetos". Los harán en todas las técnicas y en todo tipo de formas, tanto a torno como a molde, de bulto redondo o planas, acercándose a un tipo de obra que, a veces, recuerdan más a cuadros, que a cerámicas (Figura 10). La pieza presentada aquí es una pizarra esmaltada, una de las pocas firmadas por Daniel. Esta técnica era complicada, exfoliándose muchas de ellas en la cocción o posteriormente, por lo que no se debía realizar en grandes cantidades, al contrario que las cerámicas. Representa a unos tipos segovianos, mujeres campesinas (una de ellas a lomos de burro), con la iglesia de la Veracruz y la ciudad de Segovia al fondo. La escena hay que ponerla claramente en relación con la estética del 98 y su búsqueda del alma de Castilla.

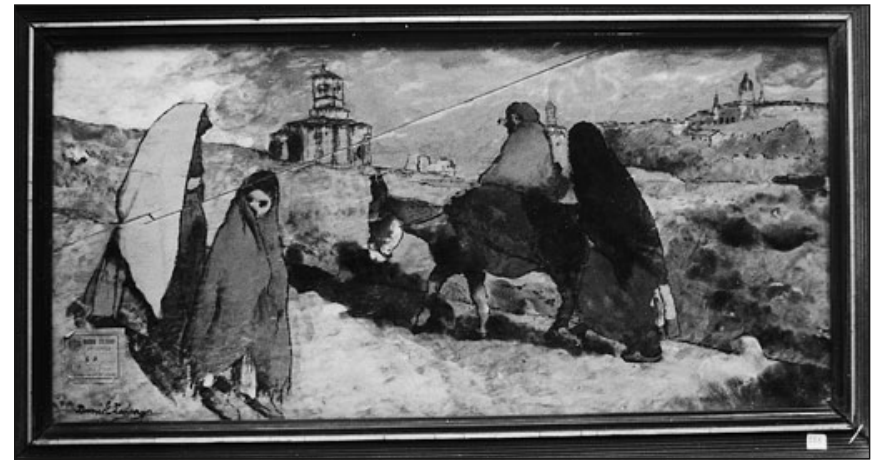

Fig. 10. Pizarra esmaltada con escena de "catetos". Daniel Zuloaga en San Juan de los Caballeros. S. XX.

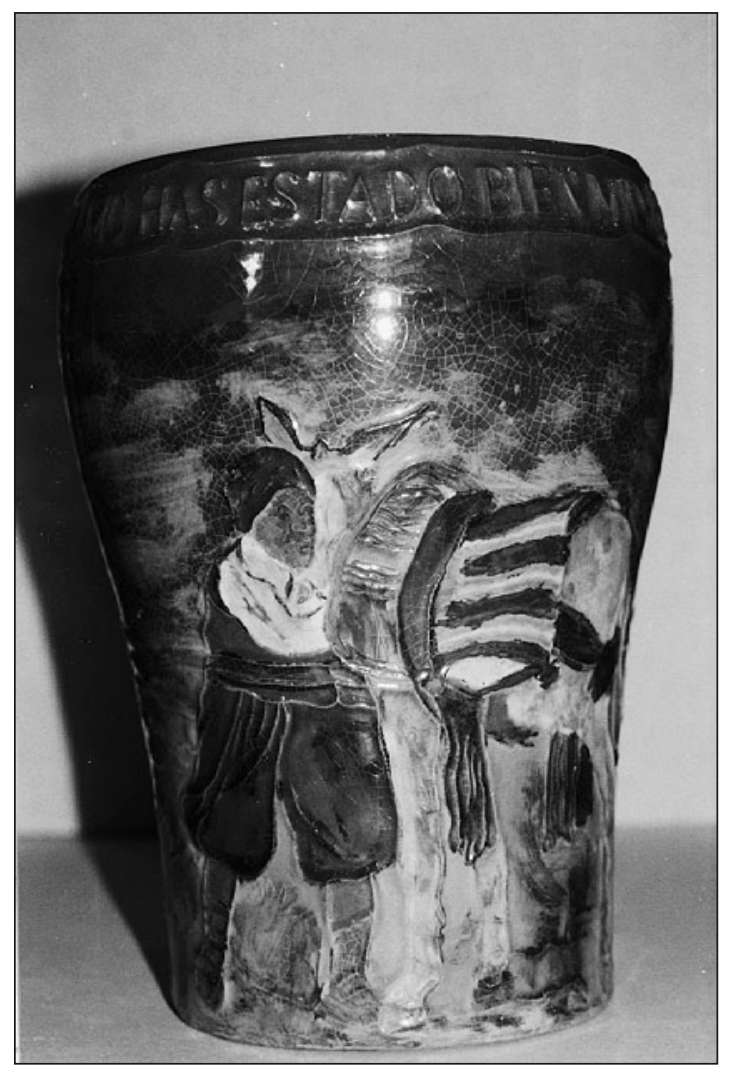

Figura 9. Tibor "Sancho". Decoración a cuenca de Sancho Panza con su rucio, copiada de una de las fotografía de Luis Ocharán de la serie del Quijote. Taller de San Juan de los Caballeros. S. XX. 


\section{BIBLIOGRAFÍA}

El Apunte Artístico. 1882. Año I. n1 10. Madrid. 30 de agosto.

"Catálogo de la Exposición Nacional de Minería, Artes Metalúrgicas, Cerámica, Cristalería y Aguas Minerales". Ministerio de Fomento. Madrid. 1883.

Catálogo de "Talleres de Hijos de Daniel Zuloaga". Segovia.

Catálogo de "Cerámica Artística para construcciones la Segoviana". Segovia.

CORESAL. 1993. "Restauración de azulejerías del Palacio de Velázquez" en Patina, n1 6. Escuela Superior de Conservación y Restauración. Madrid.

La Gaceta.1882. Madrid. 28 de enero.

“Los lunes del Imparcial”. 1922. en El Imparcial. Madrid. 14 de mayo.

P.C.A. 1916. “Exposición de Daniel y Juan Zuloaga en el Salón Parés de Barcelona. El estilo de mis cerámicas en Mercurio". Barcelona. 7 de diciembre.

A. PERLA. 1988. "Cerámica aplicada en la arquitectura madrileña". Consejería de política territorial y Dirección General de Arquitectura de la Comunidad de Madrid. Madrid.

$M^{a}$ J. QUESADA. 1984. "Daniel Zuloaga ceramista y pintor". Universidad Complutense de Madrid. Madrid.

M J. QUESADA. 1985. “Daniel Zuloaga 1852-1921”. Diputación Provincial y Caja de Ahorros y Monte de Piedad de Segovia. Segovia.

H. SCHNEIDER. 1995. "Fotocerámica". Valencia.

A. ZAMORA CANELLADA. 1998. "Museo Zuloaga. Segovia. San Juan de los Caballeros". Junta de Castilla y León. Consejería de Educación y Cultura.

J. ZULOAGA y A. MAZORRIAGA. 1934. "Castellarnau, ceramista segoviano" en Universidad y Tierra, Año I, Boletín de la Universidad Popular Segoviana. Segovia.

\section{NOTAS}

1. "El Imperial" 1922. Reproduce una nota de Daniel: "Estudiamos en Sèvres desde 1867-71, hasta que nos echaron los prusianos; fuimos allí discípulos de dos grandes químicos, messieurs Salvetat y Regnault, este último director de la fábrica de Sèvres..."

2. P.C.A. 1916 "Mercurio..." “En toda España son cientos mis obras..., pero todo a grande fuego, nunca "sur email" o pequeño fuego, que no considero como cerámica".

3. Hasta los años 80 no se conoce ninguna obra cerámica de los Zuloaga. En 1882 en la Gaceta se les presenta como “Guillermo ... cerámico, ...Germán ...escultor. ...Daniel ... pintor". De este mismo año conocemos la realización de un tapiz, reseñado en el Apunte Artístico: “Tapiz de los Sres. Zuloaga y Perea..." Está inspirado en los cartones para tapices de Goya en el asunto central, una merienda, y en la orla en aquellas de los tapices renacentistas con grutescos, putti y roleos. Fue realizado para la Cervecería Escocesa de Madrid, y al menos hacía pareja con otro, titulado en una fotografía de J. Laurent "Le Champagne", conservada en el Archivo del Museo Zuloaga.

4. Quesada, 1985. En “...los azulejos de fondo siena que flanquean el pequeño pórtico... los colores van separados con este sistema de la cuerda seca..." Pág. 78.

5. Perla. 1988. Pág. 81.

6. Coresal. 1993. Pág. 144.

7. Coresal. 1993. Pp. 144-149.

8. Se encuentra en la colección de Eleuterio Laguna de La Granja (Segovia), catalogado con el n1 472. De esta misma procedencia son el resto de las cerámicas presentadas, excepto la Figura n1 4, por lo que no repetiremos el nombre del propietario en las figuras sucesivas.

Mide $59 \mathrm{~cm}$ de altura y está firmado junto a la base en una cartela polilobulada, en blanco sobre fondo negro con "MON" y "CLOA" a uno y otro lado de una $\mathrm{H}$ y $\mathrm{Z}$ entrelazadas. En negro, debajo, a los lados "MADRID" y "1883".

9. Es una acuarela, con el dibujo al mismo tamaño que en el Tibor, firmada y fechada "D.Z. 1873".

10, "Catálogo...". 1883.

11.Su versión del jarrón de la Alhambra, realizado para la Exposición de Minería fue regalada por el rey Alfonso XII al emperador de Alemania.

${ }^{1}$.Alcántara. 1889.

“... A cargo de Guillermo ha estado la parte industrial, tierras y colores y cocción en los hornos de la Moncloa". Sin embargo, en el Archivo del Museo Zuloaga, en un nota manuscrita de Juan, se cita: "Estos paneaux de grandísimas proporciones primeramente fue ejecutados en piedra silícea de Gadacano con esmaltes estaníferos y vidriados de D.Z. pero yo niño recuerdo haciendolo en su taller de Vallehermos (Hoy donde existen las cocheras de Tranbias) no calculo bien la resistencia en plena fusion de sus hornos muflas; que sus arquetes descendieron por el peso y toda la obra se malogro, como corria prisa volvio a repetir la obra de gigantescas proporciones en azulejo pastas rojas, que por cierto como la superficie hera tan colosal la pintó en el suelo y sus riñones tubo mucho tiempo desechos de la postura, saliendo vollante como siempre este formidable hombre (yo tendría cinco años lo mas) Juan"

13. Quesada, 1985. “Una obra de este tipo ... no se intentará en Francia hasta el año 1900, en el que Sèvres se atreverá a llevar a cabo en cerámica, el gran friso pintado por Joseph Blanc para el Pabellón de Bellas Artes de París". Pág. 84.

14. Se encuentra en colección particular de la Granja (Segovia) con el n1 335, y mide $42,5 \times 22 \mathrm{~cm}$.

15.M0 Jesús Quesada la publica como de porcelana, siendo sus autores Germán y Daniel, por lo que se realizaría antes de la muerte del primero en 1886 . Antonio Perla publica que las cerámicas de la Escuela de Ingenieros de Minas se realizaron entre 1888 y 1889, leyéndose en el panel del lado Este “D. y G. Zuloaga". Perla, 1988. Pág. 84.

16.En un recorte de periódico del Archivo del Museo Zuloaga, escrito a lápiz "1886", referente a Guillermo se dice: “... Últimamente consiguió un gran triunfo, descubriendo el esmalte sobre la piedra natural, obteniendo privilegio para este invento". Si atendemos a la veracidad de esta noticia sería Guillermo y no Daniel el que inventó la manera de esmaltar piedras, aunque fuera después Daniel el que lo desarrollara, y el que se ha llevado el mérito, como vemos en un texto de Alcántara, del Catálogo de Hijos de Daniel Zuloaga: “...también presenta algunos ensayos felices de esmaltación de piedra arenisca por el estilo de la antigüedad caldeo-asiria..."

17.Quesada, 1985. Pág. 85.

18.Perla, 1988. “...Para los análisis de los materiales cerámicos he contado con la valiosa ayuda del químico ceramista Matías Castro, profesor de la Escuela Oficial de Cerámica de Madrid). Se puede afirmar que las pastas no llegaron a porcelana, lo que parece ocurrió por una falta de caolín en la misma -el color es muy amarillento-, y por una falta de grados en la cocción- aparentemente la pasta no ha llegado a vitrificar. Nos encontramos por tanto más que ante una porcelana, ante una loza dura...". Pág. 89.

19.Fórmulas en una hoja pegada en un cuaderno de Juan en el Archivo del Museo Zuloaga.

20.Se encuentra en colección particular de la Granja (Segovia) con el n1 486. Afortunadamente fue desmontada, ya que su situación de abandono, la exponía al deterioro y al pillaje, habiendo desaparecido algunos azulejos y rotos otros. Hay tres tipos de formato: cuadrado de $18 \mathrm{~cm}$. de lado, rectangular de $1,5 \times 6,3 \mathrm{~cm}$. y rectangular de $14 \times 28,5 \mathrm{~cm}$.

21.Mazorriaga, A. y Zuloaga, J. 1934. Pág. 181.

2. Esta pieza fue dada a conocer en el Congreso de la AC en 1997 en Cangas de Onís (Asturias). Se encuentra en colección particular de la Granja (Segovia), n1 287. Sus medidas son $34 \times 22 \mathrm{~cm}$. en general y $12,5 \times 9 \mathrm{~cm}$. la fotocerámica en particular.

23.Schneider, H., 1995.

24.Una fachada modernista de Zuloaga se puede ver en el n1 17 de la calle de J. Prim de San Sebastián, donde unas ninfas, mujeres desnudas, cuyas piernas terminan en aletas en lugar de pies se desenvuelven por la superficie entre tallos vegetales acuáticos. Otras fachadas modernistas han sido destruidas, como la casa de Ocharán del Paseo del Cisne, de Madrid.

25.La figura 4 está tomada de una ilustración de Mucha de la revista parisina "La plume", de 1889. Esta revista se encontraba en la biblioteca de Daniel, hoy, Archivo del Museo Zuloaga. La introducción en España de motivos modernistas, al mismo tiempo que se están produciendo en París, por artistas tan relevantes como Mucha, nos sitúa a Daniel Zuloaga como un innovador en los temas decorativos de la cerámica de su tiempo.

26. Zamora Canellada, A., 1998. Pp. 40 y 41.

27."Cerámica..." (Catálogo de la Fábrica de Vargas). "siendo nuestra primera materia Kaolin de gran blancura, podemos obtener por medio de los óxidos con que coloreamos esta pasta todos los tonos intensos y medios tonos, consiguiendo una gran intensidad en la coloración".

28.Quesada, 1985. Pág. 97.

29.Anotación manuscrita en el Museo Zuloaga, fechada el 19 de septiembre de 1921 (Daniel muere en diciembre de este año).

30.Se ha considerado a los Zuloaga muchas veces más decoradores cerámicos que ceramistas, pero la investigación de materiales está siempre presente en 
su obra. En colección particular de la Granja (Segovia) se conservan algunos azulejos con anotaciones en el reverso, como el n1 391, firmado J.Z. en el anverso, y con la siguiente fórmula en el reverso, en negro:

$\begin{array}{ll}\text { "KAOLIN } & \text { n1 27 } \\ & 0,25 \\ \text { B - O } & 0,75 "\end{array}$

Se trata de lograr una base blanca, aplicando un engobe sobre una loseta catalana industrial.

31.Además de la cerámica descrita, Juan presentó otras tres más. Se conservan las cuatro en el Museo de Artes Decorativas, y aunque no conste su procedencia, por las medidas y motivos decorativos descritos, se ajustan a la memoria presentada por Juan Zuloaga al concurso de 1934.

3. Escrito a máquina, con correcciones y añadidos a mano, en el Archivo del Museo Zuloaga:

“Materia. Los Zuloaga usan tierras segovianas mezcladas con sílice. Son principalmente de color pálido y ocres. El barro hecho con ellas se pudre casi durante un año en balsas especiales, y este barro fino se bate a mano muy detenidamente; con él se levantan en el torno a mano las piezas o se moldean los cacharros, que luego se orean al sol, y entran en el primer horno llamado de bizcocho. Cocción. Después de este primer horno de bizcocho, las piezas pasan a la esmaltación, después al segundo horno que se llama de esmalte. Aun tienen que sufrir estas piezas otra hornada, la última que se llama de reflejo...

Los hornos son de llama directa por fuego de leña de pino, que da un calor uniforme y llama muy limpia; la duración de fuego es de 12 a 18 horas".

33.Colección particular de la Granja (Segovia). Están realizadas esta pruebas sobre loseta industrial catalana de pasta roja.
34. Colección particular de la Granja (Segovia), n1 442. Medidas: 39,8 x 29,8 cm. Firmado "Juan Zuloaga", en negro, en el ángulo inferior izquierdo.

35. Podría tener relación con una nota de Juan en el Archivo del Museo Zuloaga: "También hizo mi gran padre pastas casi silíceas ...incrustadas de trozos de cuarzo, feldespato, pedernal, etc... dando a su cerámica un aspecto rudo y fuerte..."

36. Colección particular de la Granja (Segovia), n1 159. El material es arcilla ferruginosa de tono ocre claro, con una altura de $28 \mathrm{~cm}$. y un diámetro máximo de $18,2 \mathrm{~cm}$

37. Un análisis profundo de la relación entre Ignacio y Daniel, nos acercaría a no pocas influencias del segundo sobre el primero, en la elección de muchos de sus temas.

38. Entre ellas un retrato de Daniel, en bulto redondo, de $37 \mathrm{~cm}$. de altura, en bata de trabajo y con un jarrón en una mano.

39. Daniel le proporcionó modelos, objetos y antigüedades para mejor caracterizar las escenas.

40. Colección particular de la Granja (Segovia), n1 59. Arcilla ferruginosa de tono ocre claro. Medidas: Altura 22,3 cm. Diámetro máximo $16 \mathrm{~cm}$. En el solero sello impreso "DANIEL ZULOAGA".

41. Los temas de "catetos" surgen en la época de la fábrica de Vargas. En una nota de José Peña, de San Sebastián, del 3 de noviembre de 1900, una de las cerámicas es "1 plato cateto pintado".

42. Colección particular de la Granja (Segovia), n1 258. Medidas: 23 x 46,5 cm Firmado en negro "Daniel Zuloaga" en el ángulo inferior izquierdo.

43. La escena está sacada de fotografías conservadas en el Archivo del Museo Zuloaga, seguramente realizadas por Daniel Zuloaga. 